\title{
Groove Pancreatitis: A Case Report
}

Luciana Leony Valente, Mariama Alves Dantas Fagundes, Camila Medrado Pereira Barbosa, Hélio Miguel de Azevedo Bião Veiga and Maria Helena da Silva Borges

Department of Gastroenterology of Hospital São Rafael, Salvador-Bahia 41253-190, Brazil

\begin{abstract}
We report a case of groove pancreatitis in which clinical and endoscopic findings improved with conservative treatment. Most authors suggest more aggressive management, such as Whipple's surgery because of its similar findings to pancreatic carcinoma. Groove pancreatitis is a rare condition that requires more studies to establish diagnostic criteria to better classify the disease's stage and direct appropriated therapy for the patient, improving morbidity and survival of this disease.
\end{abstract}

Key words: Groove pancreatitis, pancreatic carcinoma, duodenopancreatectomy.

\section{Introduction}

Groove pancreatitis is a distinct form of chronic pancreatitis that affects the groove region located between the pancreas head, duodenum and common bile duct [1].

This condition was firstly described by french authors in 1970—Potet and Duclert—by publishing 4 cases which they described as "cystic dystrophy of the pancreas". In 1973, the "groove" term was named by Becker et al. in case series of 117 patients in Germany [2]. The prevalence of this disease is very difficult to measure because most cases are underdiagnosed, but it stays around $24.5 \%$ of patients with chronic pancreatitis [1].

Most patients who present Groove pancreatitis are males, in the ages between 40-50 years old, which predisposing factors are still unknown. It is more prevalent in those who have chronic alcoholic ingestion background [1-3]. Other associated factors described by Sánchez-Bueno et al. are: smoking, peptic ulcer and previous gastric surgical resections [2].

Clinical manifestations most common are recurrent abdominal pain which can be associated or not to nausea and vomiting. Rarely the patient can present with floating jaundice, diarrhea and diabetes [3].

Corresponding author: Luciana L. Valente, M.D., research fields: groove pancreatitis and pancreatitis.
Laboratorial test can reveal mild elevation of pancreatic and hepatic enzymes and less common concomitant elevation of serum tumor markers as CEA and CA 19.9 [4]. The disease's course is usually chronic and can take weeks to years [3].

The relevance of this disease is in the fact that its major differential diagnosis is the pancreatic head cancer which reveals a similar pattern at imaging studies. The management of these patients can be initially conservative, and most cases achieve the cure. In spite of that, when the symptoms are severe and is not possible to exclude malignant disease, surgery as Whipple's duodenopancreatectomy can be necessary [4].

The aim of this study was to describe a Groove pancreatitis case report and make a review about this disease.

\section{Case Report}

A 37-year-old man with complaints of moderated epigastralgia associated to nausea was referred to our hospital. He had consumed alcohol (about 70 g per day) for the last 20 days and had a background of chronic alcoholic ingestion for more than 10 years.

The patient had a recent past history of acute pancreatitis in the last year, after a choledocholithiasis diagnosis, and was submitted to cholecystectomy by video laparoscopy on that time. The period after the 
surgery the patient outcome complicated with recurrent episodes of acute pancreatitis in association with peripancreatic fistula and focal areas of fat necrosis of the pancreas. ERCP was performed including endoscopic papillotomy and insertion of plastic stents with improvement of clinical and laboratorial findings after this procedure.

Physical examination at admission revealed absence of jaundice, with abdominal pain at palpation of epigastrium, with no signs of peritoneal irritation. Serum pancreatic enzymes were elevated: amylase 244 U/L (normal: < $160 \mathrm{U} / \mathrm{L}$ ); lipase 2,608 U/L (normal: < $60 \mathrm{U} / \mathrm{L}$ ); liver function tests and tumor markers (carcinoembryonic antigen and carbohydrate antigen 19-9) were within the normal range. Abdominal MRI (magnetic resonance imaging) and cholangiopancreatography showed slightly enhanced in fatty peripancreatic tissue in the site of the head of the pancreas, suggesting edema and inflammation.

(A)

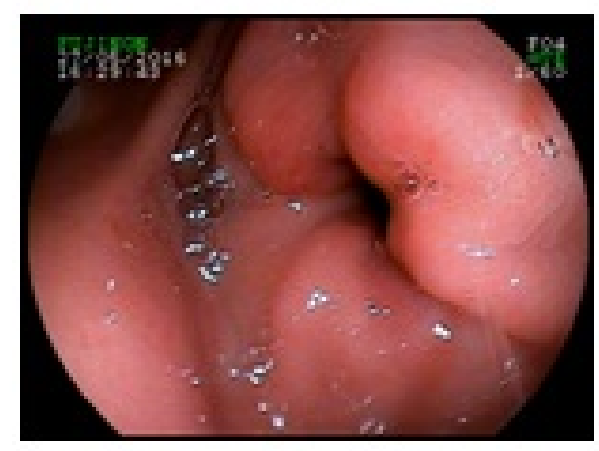

(C)

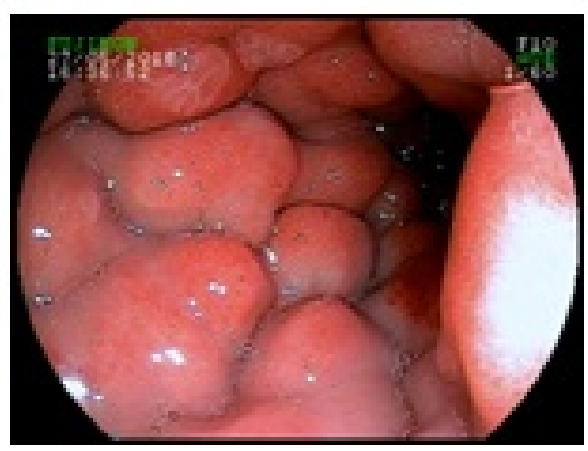

There was also a description of a persistent necrosis in the body, which measured $2.4 \mathrm{~cm}$, similar to previous imaging studies from the last year. Other findings were parietal edema in gastric antrum and duodenal bulb in addition to the other findings described.

Endoscopy was performed after those imaging findings and it revealed elevated lesions in the duodenal bulb, which raised the hypothesis of Groove pancreatitis or neoplasia (Fig. 1).

Histopathological findings from duodenal biopsies showed unspecific chronic duodenitis, which is a common pattern in cases of Groove pancreatitis.

The patient had improvement of the symptoms after clinical treatment directed to acute pancreatitis, which was based on aggressive hydration at a rate of 5 to 10 $\mathrm{mL} / \mathrm{kg}$ per hour of isotonic crystalloid solution (lactated Ringer's solution) and analgesics to pain control. It was also included in the treatment proton pump inhibitors once a day before breakfast. No more

(B)

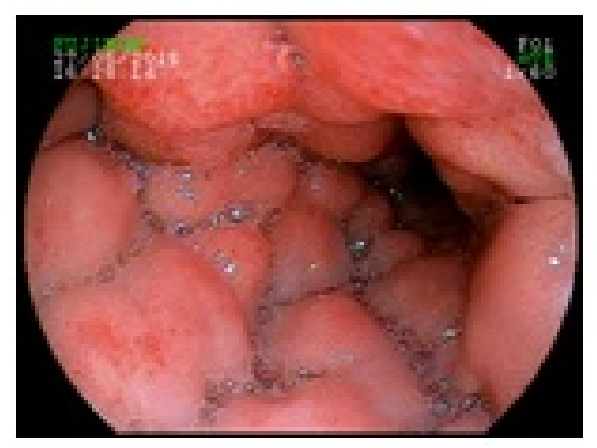

\section{(D)}

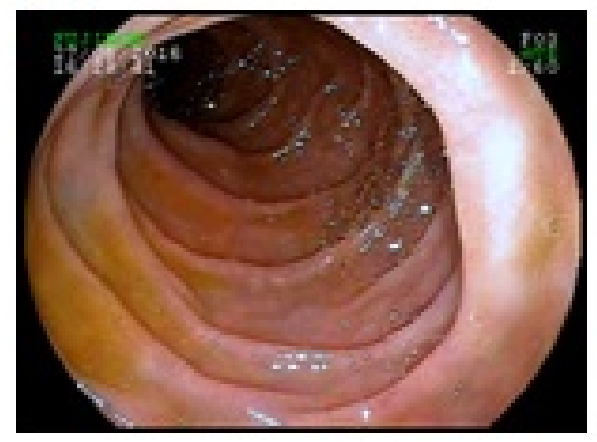

Fig. 1 From A to D: Endoscopy at admission: (A) antrum; (B) bulb 1; (C) bulb 2; (D) duodenal second portion. 


\section{(A)}

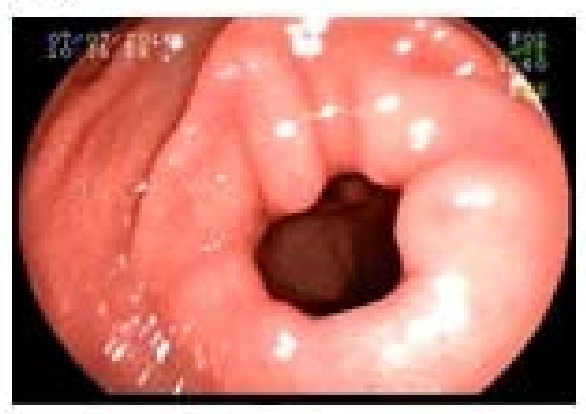

(C)

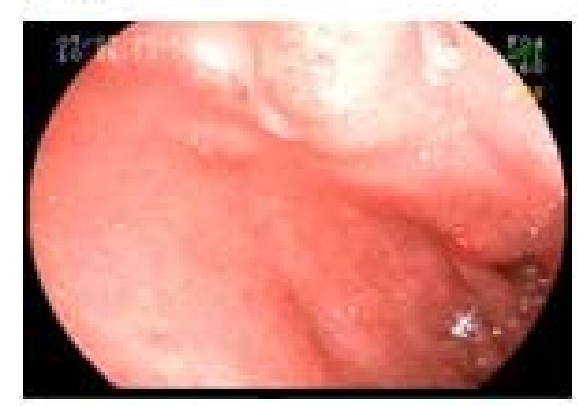

(B)

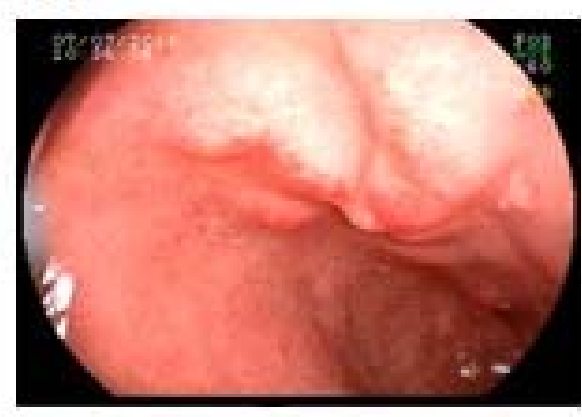

(D)

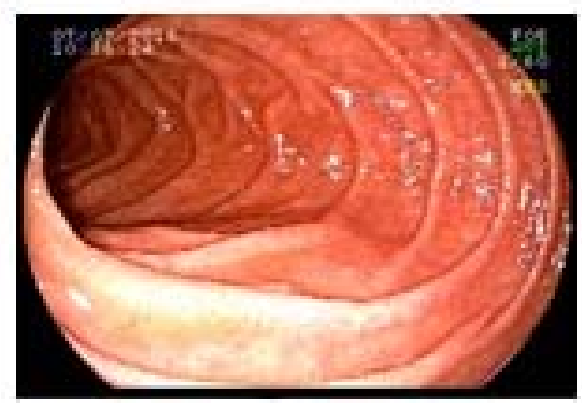

Fig. 2 From A to D: EDA before discharge: (A) antrum; (B) bulb 1; (C) bulb 2; (D) duodenal second portion.

episodes of abdominal pain, nausea or vomiting were described after $48 \mathrm{~h}$ from the beginning of the treatment. A control endoscopy was performed before his hospital discharge and it showed less number of lesions and endoscopic improvement as well (Fig. 2).

\section{Discussion}

Groove pancreatitis is a rare condition and its pathogenesis remains unclear. The first hypothesis is the anatomical or functional obstruction of the ampulla, with some abnormality in Santorini's duct. It is suggested that chronic consumption of alcohol takes to a more viscous and protein-rich pancreatic juice, which contributes to obstruction of these ducts [5]. Predisposing factors are also described by previous reports, as pancreatic heterotopias, peptic ulcers and gastric resections [2, 3].

Becker and Mischke classify Groove pancreatitis in two forms: pure and segmental. The pure form involves the groove area only, while the segmental form involves both the groove and the head of the pancreas, promoting stenosis of the pancreatic duct upstream dilatation [4].

In Groove pancreatitis it is still challenging to decide which treatment should be indicated for each patient, especially avoiding radical unnecessary surgery. Imaging studies can be helpful in differentiate the two forms of Groove pancreatitis and also from de pancreatic carcinoma.

Findings on imaging studies vary based on the stage of the disease. Abdominal ultrasonography exam in initial stages can reveal a hypoechoic band at the pancreatoduodenal Groove, which matches with inflammatory infiltration in this site. In more advanced cases, fibrosis is established and the differential diagnosis with neoplasia can be difficult. In this cases, most imaging studies shows a hyperechoic thickening of the duodenal wall. It is still possible, according to Wornski et al. to define a classic sign of the malignant disease, described as a hyperechoic pancreatic head with anechoic ducts inside [2, 3, 6].

Findings at CT scan in the pure form of the disease 
show an image of a mass between the pancreatic head and the duodenum, and most cases are also associated to cystic formations next to this area. In the segmental form, it is possible to note a dilatation of the pancreatic duct and also upstream. In the latest, the differential diagnosis remains pancreatic adenocarcinoma, which reveals very similar findings [3].

Some authors suggest that MRI should be the preferred imaging method to evaluate patient with Groove pancreatitis. Kalb et al. developed three criteria with accuracy of $87.2 \%$ to diagnose the disease, which are: focal thickening of the duodenal second portion wall; enhanced in the duodenal second portion and cystic abnormalities next to accessory pancreatic duct, with a negative predictive value to pancreatic adenocarcinoma of 92.9\% [7-9].

EUS (Endoscopic ultrasonography) is an increasing diagnostic and therapeutic method used in cases of pancreatic carcinoma, which allows acquiring tissue samples by the use of FNA (fine needle aspiration). Nevertheless, there still are not established criteria to differentiate the pancreatic distinct conditions to make a differential diagnosis with Groove pancreatitis.

In upper endoscopy, the most frequent findings of Groove pancreatitis are elevations of the mucosa in a polypoid pattern, associated or not with hyperemia or stenosis of duodenal lumen. The histopathology studies of biopsy specimens from the elevated lesions usually reveal signs of inflammation and Brunner's gland hyperplasia with no atypical cells [2, 3, 5].

ERCP

(Endoscopic

retrograde cholangiopancreatography) has less important rule in differential diagnosis of Groove pancreatitis and pancreatic neoplasia. Some findings that suggest neoplasia are irregularities of the duct caliber, which can show dilatations with or without a signal of obstruction. In Groove pancreatitis this caliber is usually regular [5].

Groove pancreatitis treatment is in most cases conservative, involving enteral nutrition, intravenous hydration and discontinues the use of alcohol and tobacco. However, some cases are recurrent, requiring surgical approach [5]. ERCP in Groove pancreatitis is useful especially in cases of bile duct obstructions which demands drainage. Most authors suggest surgery-duodenopancreathectomy

(Whipple technique) — as the treatment of choice because of the difficulties to make differential diagnosis with pancreatic carcinoma and better outcomes in relief of the symptoms compared to conservative treatment [2]. It is still also unknown the isolated influence of proton pump inhibitors on the outcome of these patients [10] because almost all cases they are used at the same time of the clinical management of acute pancreatitis.

\section{Conclusions}

The biggest challenge of Groove pancreatitis is that it still an underdiagnosed condition and requires more studies which can establish diagnostic criteria to make it possible to better differentiate with pancreatic carcinoma, in such a way that it could lead to a more appropriated therapeutic decision for the patient, improving morbidity and survival.

\section{References}

[1] Black, T. P., et al. 2014. "Groove Pancreatitis: Four Cases from a Single Center and Brief Review of the Literature.” ACG Case Rep. J. 1 (3): 154-7. (groove 4)

[2] Sánchez-Bueno, F., et al. 2016. "Groove Pancreatitis vs. Adenocarcinoma: A Review of 8 Cases.” Cir. Esp. 94 (6): 346-52. (groove 2)

[3] Pallisera-Lloveras, A., et al. 2015. "Groove Pnacreatitis.” Rev. Esp. Enfem. Diag. 2015. (groove 3)

[4] Balakrishnan, V., et al. 2007. "Groove Pancreatitis: A Case Report and Review of Literature.” JOP. J. Pancreas 8 (5): 592-7. (groove 5)

[5] Shudo, R., et al. 2002. "Groove Pancreatitis: Report of a Case and Review of the Clinical and Radiologic Features of Groove Pancreatitis Reported in Japan.” Internal Medicine 41: 537-42. (groove 1)

[6] Wornski, M., Karkocha, D., Slodkowki, M., Cebulski, W., and Krasnodebski, I. W. 2011. "Sonographic Findings in Groove Pancreatitis.” J. Ultrasound Med. 30: 111-5.

[7] Kalb, B., Martin, D. R., Sarmiento, J. M., et al. 2013. "Paraduodenal Pancreatitis: Clinical Performance of MR Imaging in Distinguishing from Carcinoma.” Radiology 
269: 475-81.

[8] Tezuka, K., et al. 2010. “Groove Pancreatitis.” Dig. Surg. 27 (2): 149-52.

[9] Raman, S. P., et al. 2013. "Groove Pancreatitis: Spectrum of Imagins Findings and Radiology-Pathology
Correlation.” Am. J. Roentgenol. 201: W29-39.

[10] Aragane, H., et al. 2006. "A Case of Recrudescent Groove Pancreatitis, Which Was in Remission by Proximal Gastrectomy with Vagotomy for Gastric Cancer.” 103 (5): $537-42$. 